\title{
Framing effects and the reversed Müller-Lyer illusion
}

\author{
JOHN PREDEBON \\ University of Sydney, Sydney, New South Wales, Australia
}

\begin{abstract}
The enclosure hypothesis of the reversed Müller-Lyer illusion was examined in three experiments. In Experiment 1, the ingoing- and outgoing-wings forms of the illusion were measured separately, as a function of the size of the gap between the ends of the shaft and the apices of the wings. In Experiments 2 and 3, the effects of a square frame and of complete and amputated versions of a rectangle on the perceived length of an enclosed horizontal line were examined. For all non-Müller-Lyer illusion figures, an inverted U-shaped function describes the relationship between illusion magnitude and the length of the test line. The peak overestimation of the test line's length was obtained when the ratio of total figure length to test line length was about 3:2. Taken together, the results of the three experiments suggest that the reversed Müller-Lyer illusion can be explained within current theoretical frameworks, such as assimilation theory, without recourse to a special factor of enclosure.
\end{abstract}

In the standard forms of the Müller-Lyer illusion shown in Figure $1 \mathrm{~A}$, the shaft of the outgoing-wings figure is perceived to be longer than the shaft of the ingoing-wings figure. However, when relatively short lines are positioned in the interwings space in the manner shown in Figure $1 \mathrm{~B}$, there is a reversal of the normal Müller-Lyer effect: the line in the ingoing-wings form appears to be longer than the physically equal line in the outgoing-wings form.

Yanagisawa (cited in Oyama, 1960) examined the reverse Müller-Lyer illusion by keeping the length of the shaft constant and displacing the fins away from the ends of the shaft. For the ingoing-wings form, the illusion changed from under- to overestimation as the distance or gap between the ends of the shaft and the apices of the wings increased, whereas for the outgoing-wings form, the illusion did not reverse but rather decreased in magnitude as a function of gap size. Fellows (1967) independently investigated the illusion by keeping the distance between the apices of the wings constant at $160 \mathrm{~mm}$ while varying the length of the shaft. The reversal of the normal Müller-Lyer effect was obtained for shaft lengths between 40 and $120 \mathrm{~mm}$, with the maximum effect occurring when the length of the line was half the interwings distance.

Fellows (1967) attributed the reversed Müller-Lyer illusion to the expansion of the shaft of the ingoing-wings form as a consequence of the enclosing or framing nature of the fins. The enclosure hypothesis was supported

This research was funded in part by a Special Projects Grant from the University of Sydney. The data of Experiments 1 and 2 were collected by Kevin Croft. Correspondence should be addressed to J. Predebon, Department of Psychology, University of Sydney, Sydney, New South Wales, Australia 2006
$\mathbf{A}$
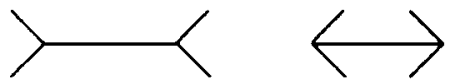

B
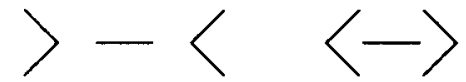

c
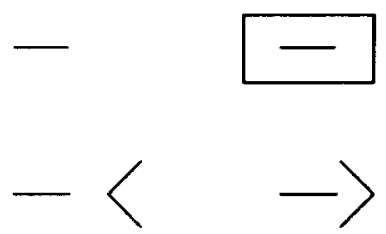

Figure 1. (A) Left- and right-hand figures are the standard outgoing- and ingoing-wings forms of the Müller-Lyer illusion, respectively. (B) The reversed Müller-Lyer illusion; (C) the rectangular frame illusion; (D) figures used by Worrall and Firth (1974).

by two findings. First, by comparing the lengths of the shafts of the two illusion forms with an unenclosed line, Fellows established that virtually the whole illusion was due to the expansionary effect of the line in the ingoingwings figure. Second, the results of a follow-up study (Fellows, 1968) indicated that a rectangular frame (Figure 1C) produced a pattern of errors that was virtually identical to the pattern of errors for the ingoing-wings Müller-Lyer figure: maximum distortion was obtained when the enclosed line was half the width of the rectangle, and the expansionary effect diminished as the length of the line increased until the illusion reversed to contraction when the enclosed line was between .75 to .9 times the width of the rectangle. Fellows interpreted the latter finding as indicating that a component of the underestimation in the standard ingoing-wings form of the MüllerLyer illusion is due to the enclosure effect of the wings. 
According to the enclosure hypothesis, the ingoing- and outgoing-wings forms of both the standard and the reversed Müller-Lyer illusions are distinct illusion types. The progressive reduction in the magnitude of the outgoing-wings illusion as a function of gap size reflects the diminution of the influence of the wings on the shaft, whereas in the ingoing-wings form, both the wings effect and enclosure influence the perceived length of the shaft. In the ingoing-wings form, shortening the shaft not only removes the influence of the wings that normally produces a contraction of the shaft's length, but also exposes the shaft to the expansionary effect promoted by enclosure.

A different explanation of the reversed Müller-Lyer illusion is provided by assimilation theories of visual illusion formation, such as Brigell, Uhlarik, and Goldhorn's (1977) neural coding model and Pressey's (1967; Pressey $\&$ Bross, 1973) attentive field theory. For example, Pressey's theory asserts that the perceived lengths of the shafts in the normal forms of the Müller-Lyer figures (Figure 1A) become assimilated to the lengths of the implied contextual magnitudes produced by the fins. Since the contextual magnitudes are all either longer or shorter than the shaft of the outgoing- or ingoing-wings forms, respectively, the shaft's length will be correspondingly overor underestimated. The effect of introducing a gap between the shaft and the fins in the ingoing-wings figure is to change the relationship between the contextual magnitudes and the shaft: as the gap increases, there will be a point at which all the contextual magnitudes will be longer than the shaft, and, hence, the perceived length of the shaft will be assimilated toward the longer contextual elements, the effect of which is to reverse the ingoingwings illusion from underestimation to overestimation. According to assimilation theory, then, both the reversed and the standard forms of the Müller-Lyer illusions are promoted by similar illusion-inducing processes (Jordan \& Uhlarik, 1986; Pressey, \& Bross, 1973).

Although Pressey's account of the reversed Müller-Lyer illusion is consistent with the results of a number of studies (Pressey \& Bross, 1973; Pressey \& Di Lollo, 1978; Pressey, Di Lollo \& Tait, 1977), two aspects of Fellows's $(1967,1968)$ findings pose problems for assimilation theory. First, the enclosure hypothesis is given credence by the remarkable similarity of the inverted U-shaped function relating illusion magnitude to the length of the shaft of the ingoing-wings figure, on the one hand, and to the length of a line enclosed in a rectangular frame, on the other. Second, assimilation theory cannot explain the reversal from expansion to contraction when the length of the line is greater than about .75 of the width of the rectangle: since the contextual magnitudes are longer than the enclosed line, only expansion is predicted by the theory.

Fellows's $(1967,1968)$ enclosure hypothesis relies crucially on the results of his rectangle experiment. However, different results have been obtained with other figures that might be expected to implicate an enclosure effect. Kün- napas (1955) assessed the effect of a square frame on the perceived length of an enclosed horizontal line of constant length and found a direct function whereby illusion magnitude was related to the size of the square. Similarly, Thorn (1973; cited in Fellows, 1973), in a study similar to Künnapas's, examined the independent contributions of the vertical lines and of the horizontal lines (see Figures $2 \mathrm{E}$ and $2 \mathrm{~F}$ ) of rectangles of different sizes to the perception of a center line of constant length: rather than an inverted U-shaped function, there was consistent reduction in the expansionary effect on the perceived length of an "enclosed" line as a function of the length of flanking horizontal lines (Figure 2E) and of the distance between the vertical lines and the center line (Figure 2F).

Despite its obvious importance for theories of the Müller-Lyer illusion, the reversed illusion form has received remarkably little empirical scrutiny. One aim of the present investigation, therefore, was to obtain further information on the reverse Müller-Lyer illusion. In part, it was motivated by Worrall and Firth's (1974) surprising findings. Using the single-wing figures shown in Figure 1D, they indirectly assessed the pattern of length distortions by measuring the apparent position of the midpoint of the line as a function of its proximity to the fins. Contrary to both Yanagisawa's (cited in Oyama, 1960) and Fellows's (1967) results, Worrall and Firth concluded that the major factor in the reverse illusion was the outgoing-wings and not the ingoing-wings figure. With the exception of the standard Müller-Lyer forms, for both the ingoing- and the outgoing-wings figures the perceived length of the shaft as a function of gap size was underestimated, with the underestimation more pronounced for the outgoing-wings than for the ingoing-wings figure when the length of the line was at a certain minimum length. Worrall and Firth's results are puzzling and, if confirmed, pose a fundamental challenge to current theoretical treatments of the Müller-Lyer illusion. For this reason, in Experiment 1 the magnitudes of the length distortions were examined separately in the two forms of the Müller-Lyer illusion as a function of the gap between the shaft and the fins.

The present investigation was also an attempt to evaluate the enclosure hypothesis. A number of studies (e.g., Brigell \& Uhlarik, 1979; Brigell et al., 1977; Schiano, 1986) have shown that the magnitudes of many illusions of extent are influenced by the framing ratio-that is, the ratio of the total length of the illusion figure to the length of the test line-with maximum overestimation occurring at a framing ratio of about 3:2. Since such inverted Ushaped functions describe the pattern of length distortions in illusion figures, such as the divided-line illusion (Brigell et al., 1977), for which the notion of enclosure seems inapplicable, Fellows's (1968) rectangle results may not be due to an enclosure effect at all, but rather to a framing effect. This issue was investigated in Experiments 2 and 3 , in which the effects of a square frame and of complete and amputated versions of a rectangle on judgments of the length of an enclosed horizontal line were examined. 


\section{EXPERIMENT 1}

As in Fellows's (1967) study, in Experiment 1 shaft length was varied while the distance between the apices of the wings was kept constant. However, the relative lengths of the outgoing- and ingoing-wings forms were not assessed; instead, the magnitudes of the two illusion forms were measured separately. Such a design permits the reversed Müller-Lyer illusion to be evaluated from two different frameworks. When the two illusion forms are considered separately, the reversed illusion usually refers (see, e.g., Pressey \& Bross, 1973) to the change from under- to overestimation of the length of the shaft of the ingoing-wings form, the assumption being that reversal from over- to underestimation does not occur for the outgoing-wings form. Such an approach obviously does not address the issue of the relative magnitudes of the two illusion forms: even if the ingoing-wings form is overestimated, this overestimation might still be smaller than the overestimation of the outgoing-wings figure. From this perspective, a reversed illusion occurs only when the illusion magnitude of the ingoing-wings figure is greater than that of the outgoing-wings figure.

\section{Method}

Subjects. The subjects were 24 undergraduate students drawn from an introductory psychology course. All had emmetropic or corrected vision.

Stimuli. There were three sets of stimulus figures; the ingoingand outgoing-wings Müller-Lyer forms and a plain control line (Figures $2 \mathrm{~A}, 2 \mathrm{~B}$ and $2 \mathrm{C}$, respectively). All lines were approximately $.5 \mathrm{~mm}$ wide. The internal angle of the wings formed an angle of $60^{\circ}$. The lengths of the oblique lines and the distance between the vertices of the wings were 30 and $80 \mathrm{~mm}$, respectively.

There were $\mathbf{1 0}$ figures in each stimulus set. Each set of figures differed only in the length of the horizontal test line. The lengths of these lines were $10,20,30,40,50,60,65,70,75$ and $80 \mathrm{~mm}$ to span the $80-\mathrm{mm}$ distance between the wing vertices. For the ingoing- and outgoing-wings figure series, the horizontal line was

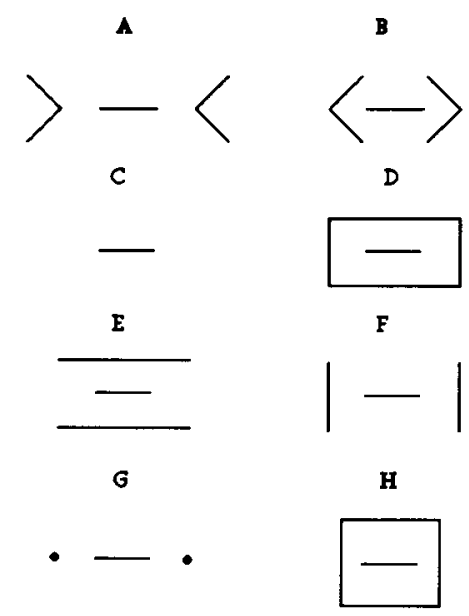

Figure 2. Figures used in Experiments 1 (A, B, C), 2 (B, C, D, $E, F, G)$, and $3(C, D, H)$ : Figures $A$ and $B$ are the outgoing- and ingoing-wings Müller-Lyer figures, respectively; $C$, control line; $D$, rectangle; $E$, horizontal lines; F, vertical lines; G, dot-line; H, square. positioned symmetrically with respect to the midpoint of the distance between the vertices. Each figure was drawn separately in black ink on white cardboard sheets.

Apparatus. The apparatus was similar to the one used by Predebon (1983). It consisted of a white test and a white comparison cardboard field. The test field was tilted back from vertical at $45^{\circ}$, and the comparison field was positioned horizontally, meeting the test field at $135^{\circ}$. The subject, with chin resting on a chinrest, viewed the comparison and test fields from a distance of $45 \mathrm{~cm}$ under normal fluorescent room illumination.

The stimulus cards were slotted behind the test field, with the figure centered in a $20-\mathrm{cm}$-diam circle cut out in the test field. The comparison line was a black horizontal line, $12 \mathrm{~cm}$ long and $.5 \mathrm{~mm}$ wide, drawn on white paperboard, and it was viewed through an ellipsoid cut out (major and minor axes of $30 \mathrm{~cm}$ and $20 \mathrm{~cm}$, respectively) in the comparison field. A white rigid occluding panel was slotted between the comparison field and the surface on which the comparison line was drawn. By grasping the ends of the occluding panel, which protruded beyond the edges of the comparison field, the subjects could move the panel laterally, and hence, by covering or uncovering the length of the comparison line, they could vary its length. Neither the guides between which the panel moved nor the scale that permitted measurements of the subjects' adjustments to the nearest $.5 \mathrm{~mm}$ was visible to subjects.

Procedure. The subjects' task was to reproduce the apparent length of the test line of the stimulus figures. Two judgments were made for each figure: one judgment was made with the comparison line set initially at a noticeably longer extent than the test line, and the other with the comparison line set noticeably shorter. Bracketing was permitted. After each judgment, the subject turned away from the stimulus field while the experimenter recorded the setting and replaced the stimulus card for the next trial. There were three blocks of 10 trials, with only one stimulus set series presented in each block. The three blocks were randomized across subjects as was the presentation of the 10 figures in each block. Thus, each subject made a total of 60 judgments, two adjustments for each of the 10 figures in each of the three blocks. All subjects completed the two adjustments for a given figure before proceeding to the next figure, and there was a break of 5 min between each block of trials.

\section{Results and Discussion}

The subjects' two adjustments of each figure were averaged. The subjects consistently overestimated the lengths of the control lines. This overestimation or, equivalently, underestimation of the length of the response line ranged from an average of $1.8 \mathrm{~mm}$ for the $80-\mathrm{mm}$ control line to an average of $.16 \mathrm{~mm}$ for the $20-\mathrm{mm}$ control line. The length judgments of the 10 shaft lengths of each of the two Müller-Lyer figure series were converted to deviations from the point of subjective equality (PSE) by subtracting from each judgment of the shaft the judgment of the corresponding control line. Since the shafts varied in physical length, these deviations were normalized as a percentage of the actual length of the shaft. Figure 3 shows the mean percentage illusion for both illusion forms as a function of the length of the test line expressed as a percentage of the full shaft length, with positive and negative scores indicating over- and underestimation of the length of the test line relative to judgments of the length of the appropriate control line. The standard (i.e., no gap between shaft and vertices) Müller-Lyer forms are represented by the $100 \%$ abscissa value.

As is shown in Figure 3, increasing the length of the shaft or, equivalently, decreasing the gap between the 


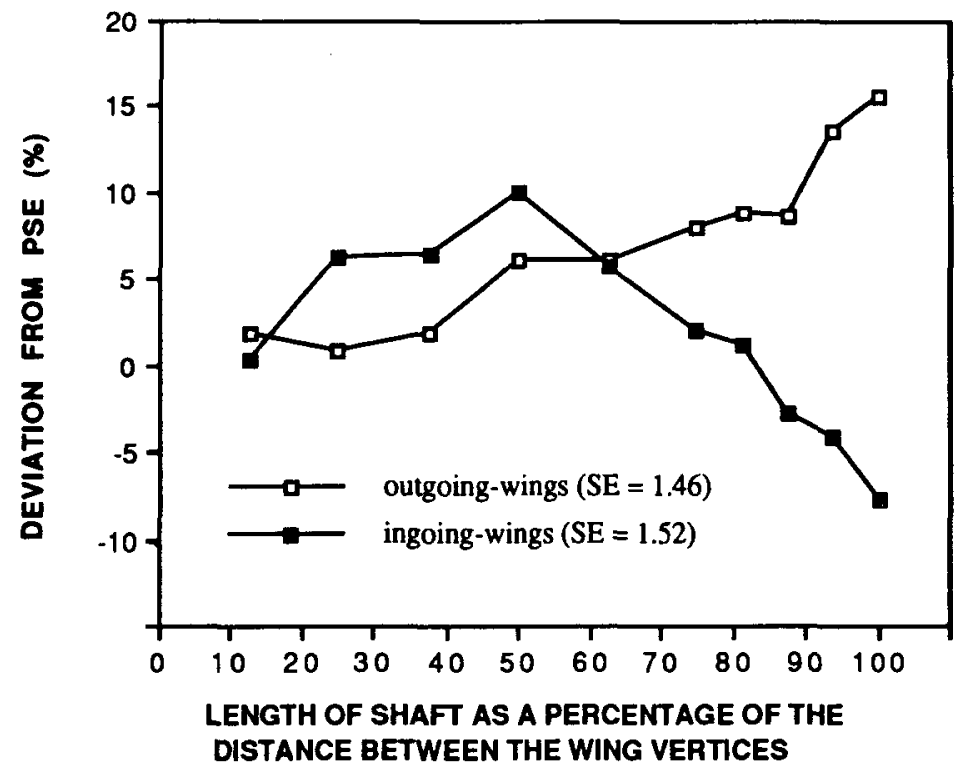

Figure 3. Mean percentage deviations from the point of subjective equality as a function of the length of the shaft, expressed as a percentage of the 80-mm distance between the wing vertices of the ingoing- and outgoing-wings forms of the Muiller-Lyer figure. The average standard errors $(S E)$ of the 10 measurement points of each condition are shown in the legend. Note that the illusory effect obtained with the full-length shaft reverses as shaft length is reduced for the ingoing-wings form but not for the outgoing-wings form (Experiment 1).

shaft and the wings has different effects on the two illusion forms. In the case of the ingoing-wings figure, the standard illusion (i.e., underestimation) reverses to overestimation when the length of the shaft is less than about $80 \%$ of the distance between the wing tips, and the overestimation increases to a maximum when the shaft is approximately half the interwing distance, after which the illusion decreases. In contrast, for the outgoing-wings illusion, the standard illusion (i.e., overestimation) does not reverse, but rather its magnitude decreases as a function of the length of the shaft. The outcomes of two-way analysis of variance (ANOVA) performed on the data confirm these observations: the main effects of illusion type $[F(1,23)=57.05, p<.05]$ and shaft length $[F(9,207)=2.84, p<.05]$ were significant, as was their interaction $[F(9,207)=34.85, p<.05]$. As a means of describing the data, separate trend analyses were performed on the data of the two conditions. Only the linear trend component was significant in the analysis of the outgoing-wings data $[F(1,207)=82.05, p<.05]$, while the linear, quadratic, and cubic trend components were significant in the analysis of the ingoing-wings data $[F(1,207)=91.55,54.76$, and 14.32 , respectively, $p<.05]$.

The present findings are consistent with both definitions of the reversed Müller-Lyer illusion. On the one hand, if the two Müller-Lyer forms are considered separately, a reversal of the normal illusion occurs only in the ingoing-wings form, whereas the effect of increasing the gap size simply decreases the magnitude of the outgoing- wings illusion. On the other hand, Figure 3 shows that for test line lengths of $25 \%-50 \%$ of the full length shaft, the ingoing-wings illusion is not only positive but its magnitude is also greater than the outgoing-wings illusion. This relationship is, of course, the reverse of the normal Müller-Lyer effect.

These results are inconsistent with Worrall and Firth's (1974) claim that the major factor in the reverse illusion is the outgoing-wings figure. Furthermore, and contrary to their findings, a gap between the shaft and vertices in the outgoing-wings figure does not produce a perceptual underestimation of the shaft's length.

\section{EXPERIMENT 2}

The enclosure hypothesis is given credence by Fellows's (1968) finding that a rectangular frame and the ingoingwings Müller-Lyer figure produce virtually identical inverted U-shaped error functions. However, as noted previously, such functions have also been obtained with certain illusion configurations for which the role of enclosure seems inapplicable. This observation implies that inverted U-shaped functions are not specific to figures possessing the property of enclosure. If so, the amputated versions of the rectangle shown in Figures $2 \mathrm{E}$ and $2 \mathrm{~F}$ should produce a similar pattern of errors as a rectangular frame (Figure 2D).

The two amputated versions of the rectangle are relevant to assimilation models of the reverse Müller-Lyer illusion. According to assimilation theory, these figures 
have contextual magnitudes similar to those of the complete rectangle and, therefore, all three figures should produce similar error functions. However, whereas Fellows (1968) reported an inverted U-shaped function for a line enclosed in a rectangle, Thorn (1973; reported in Fellows, 1973) found a consistent reduction in the illusory effect as the size of the amputated rectangles increased (Figures $2 \mathrm{E}$ and $2 \mathrm{~F}$ ). Although these findings might suggest that the rectangular frame and the ingoing-wings figure have a common feature (a global closure effect?) that is not present in the amputated versions of the rectangle, in view of the structural similarity of the amputated and complete rectangular figures it seemed prudent to reexamine these illusions. The main purpose of this experiment, therefore, was to assess in the one design the similarity or otherwise of the patterns of errors with the ingoing-wings Müller-Lyer figure and with the complete and amputated rectangular figures. The ingoing-wings figure was included not only as an independent check of the findings of Experiment 1 but also to determine whether the rectangle and ingoing-wings figures produce virtually identical error functions, as is suggested by the results of Fellows' $(1967,1968)$ experiments. A minimal form of the rectangle figure-namely, the dot-line figure (Figure 2G) - was also included, in order to examine whether or not discrete contextual contours are necessary to produce inverted $U$-shaped error functions.

\section{Method}

The six stimulus set figures used in this experiment are shown in Figures $2 B-2 G$. The widths of all the lines were approximately $.5 \mathrm{~mm}$. The ingoing-wings figure was identical to that of Experiment 1 , and the dimensions of Figures 2D-2G were derived from this figure. Thus, the distance of $80 \mathrm{~mm}$ between the vertices of the ingoing-wings figure matched the width of the rectangle (Figure 2D), the lengths of the flanking horizontal lines in Figure 2E, the distance between the vertical lines in Figure $2 \mathrm{~F}$, and the distance between the dots in Figure 2G. Similarly, the $30-\mathrm{mm}$ vertical distance between the wing tips of the ingoing-wings figure matched the length of the vertical sides of the rectangle, the vertical lines in Figure $2 \mathrm{~F}$, and the vertical separation between the flanking lines in Figure 2E. The diameter of the flanking dots in Figure $2 \mathrm{G}$ was $2 \mathrm{~mm}$. There were eight figures in each of the six stimulus sets. These figures differed only in the length of the central test line: 10, 20,30,40,50,60,70, and $80 \mathrm{~mm}$. There were six blocks of trials, with only one stimulus series presented in each block. The six blocks were randomized across subjects, as was the presentation of the eight figures in each block. Thus, each subject made a total of 96 judgments, two adjustments of each of the eight figures in each block. The 16 subjects all had normal or corrected vision, and none had participated in the previous experiment. In all other respects, the procedure was similar to that of Experiment 1.

\section{Results and Discussion}

Subjects overestimated the lengths of the control lines, and their average overestimation ranged from $2.12 \mathrm{~mm}$ for the $80-\mathrm{mm}$ line to $.47 \mathrm{~mm}$ for the $10-\mathrm{mm}$ line. As in Experiment 1, judgments of the five noncontrol stim-

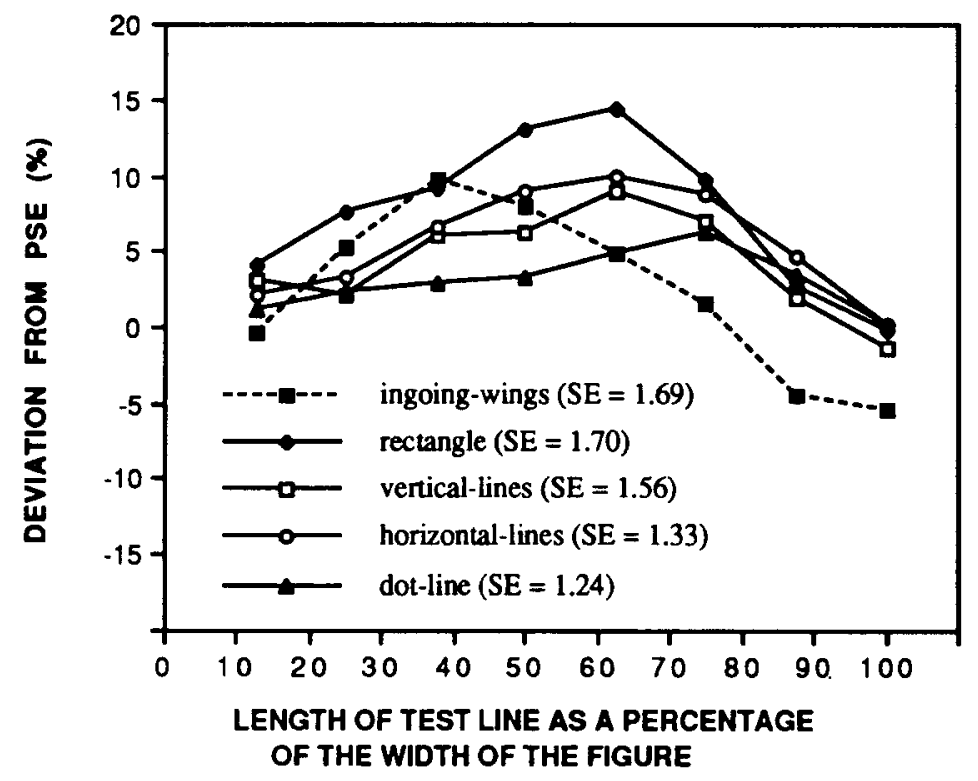

Figure 4. Mean percentage deviations from the point of subjective equality as a function of the length of the test line, expressed as a percentage of the $80-\mathrm{mm}$ long figures. The average standard error (SE) of the eight measurement points of each condition are shown in the legend. Note that for all four non-Müller-Lyer figures, the pattern of errors describes an inverted U-shaped function with maximum illusory effects occurring for test line lengths of approximately $63 \%$ and $75 \%$ of the total width of the figures (Experiment 2). 
ulus set series were converted to deviations from the PSE by subtracting judgments of the control lines from judgments of the corresponding test lines, and these deviation values were normalized by dividing each score by the actual length of the test line.

Figure 4 shows the mean errors as a function of the length of the test line, expressed as a percentage of the overall width $(80 \mathrm{~mm})$ of the test figures, for each of the five stimulus series. The results of the two-way ANOVA performed on these data yielded significant main effects of figure type $[F(4,60)=17.44, p<.05]$ and line length $[F(7,105)=9.76, p<.05]$ and a significant interaction $[F(28,420)=4.52, p<.05]$; as is evident in Figure 4, the interaction reflects the differential effect of the length of the test line on the patterns of errors across the five conditions and, in particular, the difference between the ingoing-wings condition and the other four conditions.

Separate trend analyses were performed on the data of each of the five conditions. The inverted U-shaped character of the error functions was confirmed by the significant quadratic trend component in the analyses of the data of all five conditions $[F(1,105)=8.50,48.35,11.25$, 38.26 , and $25.22, p<.05]$. The only other significant trends were the linear component in the analysis of the rectangle data $[F(1,105)=5.27, p<.05]$ and the linear and cubic trend components in the analysis of the ingoingwings data $[F(1,105)=25.64$ and $8.15, p<.05]$.

The major findings can be summarized as follows. First, the results of the ingoing-wings condition are generally consistent with those of Experiment 1. Although the peak overestimation occurred at different line lengths (i.e., $37.5 \%$ vs. $50 \%$ of the full shaft length), the illusion reversed from underestimation to overestimation at line lengths between $75 \%$ and $87.5 \%$ of the full shaft length, and the magnitudes of the peak overestimation were similar. Second, all four non-Müller-Lyer conditions produced an inverted U-shaped function that, with the exception of the dot-line figure, peaked at approximately $63 \%$ of the full figure width-that is, at a ratio of total figure length to test line length of $1.6: 1$. Third, contrary to Fellows's $(1961,1968)$ finding, it is obvious from Figure 4 that the rectangle and ingoing-wings figures did not produce an identical pattern of expansion and contraction of the test line. The peak expansion of the line occurred at different line lengths and, importantly, there is no evidence here that the expansionary effect of the rectangle reversed to contraction as the length of the line increased or, equivalently, as the gap size decreased. Fourth, the results of the dot-line condition indicate that neither a rectangular frame nor its vertical or horizontal components are necessary to produce an expansion effect.

\section{EXPERIMENT 3}

Although the inverted U-shaped error function of the rectangle figure condition of Experiment 2 partially confirms Fellows's (1968) findings, it is inconsistent with the results of Künnapas (1955) and of Thorn (1973; reported in Fellows, 1973), both of whom found that the expansionary effect of a rectangular frame decreased systematically as a function of frame size. Perhaps this discrepancy reflects methodological differences. Both Künnapas and Thorn kept the length of the test line constant, whereas in Experiments 1 and 2, following Fellows (1967), I used the unorthodox procedure of varying the length of the test line. The purpose of this experiment was to reexamine the effects of both rectangular and square frames on an enclosed line. Unlike Experiments 1 and 2, however, in this experiment, the test line remained constant while the width of the frames was varied.

\section{Method}

The stimulus figures are shown in Figures $2 \mathrm{D}$ and $2 \mathrm{H}$. There were two sets of stimuli, each consisting of seven test stimuli and three distractor stimuli. For one set, the horizontal test line was positioned in the center of a square frame; for the other set, it was positioned in the center of a rectangular frame. Each stimulus was printed in black ink and centered on the left-hand side of a 29.5 $\times 21 \mathrm{~cm}$ sheets of white paper. All lines were approximately $.5 \mathrm{~mm}$ wide. The vertical separation of the horizontal lines of the rectangle was always $13 \mathrm{~mm}$. The widths of the squares and of the rectangles were $81,55,43,33,27,21$, and $18 \mathrm{~mm}$, and the test line was always $18 \mathrm{~mm}$ long. For the three distractor stimuli, the length of the enclosed line was 10,14 , or $22 \mathrm{~m}$. A $33-\mathrm{mm}$ horizontal line was positioned on the right-hand side of the stimulus sheet and aligned with the test line. The distance between the midpoint of the test line in the square frame and the left-hand point of the response line was fixed at $90 \mathrm{~mm}$. In order to familiarize the subjects with the task, there were three practice stimuli, each consisting of a single horizontal line of 12,18 , or $23 \mathrm{~mm}$.

There were three groups of subjects. For reasons not relevant here, two groups judged the square frame stimuli. The procedures for these two groups were identical, except that one group $(n=9)$ made judgments of all seven test stimuli (Square Series B), while for the other group $(n=11)$ the 18- and 81 -mm square frames were not included in the test series (Square Series A). The third group ( $n=12$ ) judged the rectangular frame stimuli.

The test sheet was positioned so that its long side was aligned to the edge of a small desk in front of the seated subject. The subject's task was to reproduce the perceived length of the horizontal line of the test figure or, in the case of the practice figures, the perceived length of the single line, by placing a mark through the response line so that the distance between the mark and the lefthand point of the response line appeared equal to the test line's length. The subjects were permitted to reposition the mark if necessary, although very few did so. After completing a given judgment, the experimenter replaced the test sheet for the next judgment. All observers were first given the three practice stimuli followed by the random presentation of the seven or five test and three distractor stimuli. After a 30-sec break, the procedure was repeated with a new random order of the practice, test, and distractor stimuli. The subjects viewed the stimuli without a chin or head restraint from a distance of approximately $45-60 \mathrm{~cm}$ under normal fluorescent room illumination. None of the 32 subjects had participated in the previous experiments, and all had normal or corrected vision.

\section{Results and Discussion}

The judgment of the 18-mm practice line was used to determine the PSE of the test stimuli. The mean reproductions of the $18-\mathrm{mm}$ line were 18.8 and $18.3 \mathrm{~mm}$ for the two square figure conditions and $18.1 \mathrm{~mm}$ for the rectangular figure condition. Judgments of the $18-\mathrm{mm}$ hori- 


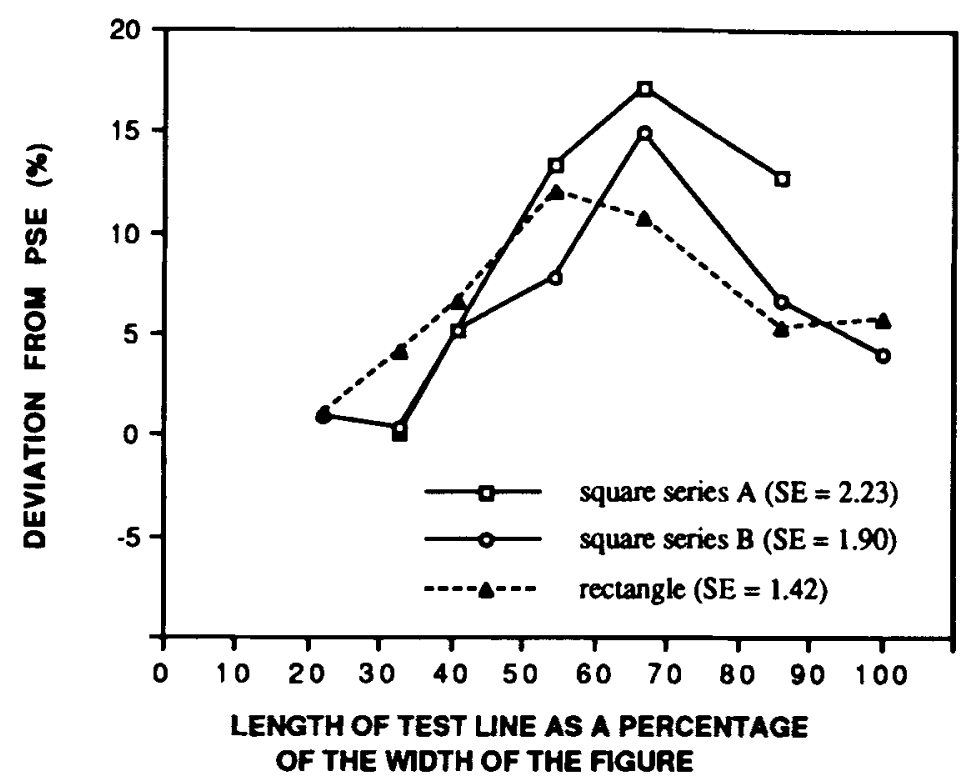

Figure 5. Mean percentage deviations from the point of subjective equality as a function of the length of the 18-mm-long test line, expressed as a percentage of the width of the rectangle (seven frames), Square Series $A$ (five frames), and Square Series B (seven frames) figures. The average standard errors (SE) of the measurement points of all three conditions are shown in the legend. Note that for all three figures, an inverted U-shaped curve describes the error functions, with maximum illusory effects occurring when the lengths of the test line are $67 \%$ and $55 \%$ of the width of the square and rectangle frames, respectively (Experiment 3).

zontal line in the test stimuli were converted to deviations from the PSE by subtracting the PSE from the mean judged length for each test stimulus. To facilitate comparisons with the results of Experiments 1 and 2, the deviation scores were divided by the length of the test line $(18 \mathrm{~mm})$; these data are shown in Figure 5 for each of the three conditions as a function of the length of the test line, expressed as a percentage of the width of the figures.

As can be seen in Figure 5, an inverted U-shaped curve describes the error functions of all three conditions, with peak overestimation occurring when the $18-\mathrm{mm}$ test line was $66.7 \%$ and $54.5 \%$ of the width of the square and rectangle figures, respectively, or, equivalently, for figure width to line length ratios of $1.5: 1$ and $1.8: 1$, respectively. The change in the magnitude of the errors as a function of the figure's width was confirmed by the significant oneway ANOVA performed separately on the error data of the two square figure conditions $[F(6,48)=7.13$, $p<.05 ; F(4,40)=7.74, p<.05]$ and the rectangle figure condition $[F(6,66)=4.15, p<.05]$.

The present findings are generally consistent with the results of the rectangle condition of Experiment 2. Both produced inverted $U$-shaped error functions with the maximum distortion occurring at similar framing ratios (1.5:1 to $1.8: 1$ ). This replication is particularly noteworthy in view of the marked differences in the size of the stimuli and in the measurement procedure. Moreover, these findings suggest that Künnapas's (1955) and Thorn's (cited in Fellows, 1973) finding of a systematic decrease in the expansionary effect of the frame as a function of its width cannot be attributed to the procedure of using a test line of constant length.

\section{GENERAL DISCUSSION}

There are three principal findings of the present investigation. First, the reversed Müller-Lyer illusion is determined mainly by the ingoing-wings form: reversal of the illusion occurred for the ingoing-wings but not for the outgoing-wings form. Second, the rectangle figure and the ingoing-wings Müller-Lyer figure did not produce equivalent patterns of expansion of the test line's length: maximum overestimation occurred at a different framing ratio, and there is no evidence that the expansionary effect of the rectangle reverses to contraction as the length of the test line approaches the width of the rectangle. Finally, the inverted $U$-shaped function relating illusion magnitude to the length of the test line is not specific to the rectangle figure. It occurs with a variety of contextual elements, including the limiting condition represented by the dot-line figure.

The initial appeal of the enclosure hypothesis stemmed from Fellows's $(1967,1968)$ findings of a remarkable similarity in the shape and absolute magnitudes of the error functions produced by the ingoing-wings Müller-Lyer figure and by a rectangular figure of equivalent dimensions. The results of the present investigation, however, indicate that the ingoing-wings and rectangle figures do not produce identical patterns of distortions. In particular, the expansionary effect of the rectangular frame does not re- 
verse to contraction as the length of the enclosed line approaches the width of the rectangle.

Nevertheless, it might be argued that the expansionary component of the ingoing-wings figure is sufficiently similar to the inverted $U$-shaped function of the rectangle figure to implicate an enclosure effect (see Figure 4). Two considerations question the plausibility of such an interpretation, however. First, rather than invoke an enclosure mechanism, all the effects reported here can be described in terms of the principle of perceptual assimilation-that is, as a shift of the perceived length of the test line to the lengths of the contextual extents. According to current models of assimilation, the difference in the absolute magnitudes and in the patterns of errors between the ingoingand outgoing-wings Müller-Lyer forms (Experiment 1) and between the complete and amputated versions of the rectangle figure (Experiment 2) can plausibly be attributed to the differential effectiveness of the contextual elements in defining the explicit and implicit contextual magnitudes of a particular stimulus configuration. Second, the results of Experiments 2 and 3 show that for both the rectangle and the square figures, maximal distortions occur for framing ratios approaching 1.6:1. This value is similar to the framing ratio at which peak overestimation is obtained in figures, such as the divided-lines illusion (see, e.g., Brigell et al., 1977), for which the notion of enclosure is inapplicable.

Three additional issues raised by the present findings warrant brief comment. First, Fellows's (1967) reversed Müller-Lyer findings occasionally have been cited as supporting the claim that the ingoing- and outgoing-wings forms of the Müller-Lyer illusion are distinct illusion types. The present findings, however, suggest that it might be unnecessary to invoke different causal mechanisms: rather, they support assimilation theory's unitary explanation of both the standard and the reversed Müller-Lyer illusions. In particular, whereas the markedly different error functions of the rectangular and the ingoing-wings figure illusions question the plausibility of the enclosure hypothesis of the reversed Müller-Lyer illusion, they are consistent with assimilation theory's account of the influence of contextual extents on judgments of the length of a focal extent. Second, the present results contradict Worrall and Firth's (1974) finding that the effect of introducing a gap is to contract the perceived length of the lines in both forms of the Müller-Lyer illusion. This discrepancy may reflect a fundamental incongruity between judgments of length and judgments of the apparent midpoint of a line, analogous to the demonstrated incongruity between the apparent position of the vertices and the apparent lengths of the shafts in Müller-Lyer figures (Gillam \& Chambers, 1985; Mack, Heuer, Villardi, \& Chambers, 1985). Finally, a few studies (see Oyama, 1960; Pressey, 1988) have yielded length contrast-that is, underestimation of a test line's length when its length is much shorter than the lengths of the contextual extents. In none of the experiments reported here, however, did length contrast occur with the shorter test lines. Kolbert and Sarris (1984), using a modified MüllerLyer figure, also failed to find contrast for line lengths ranging from $20 \%$ to $55.8 \%$ of the full length of the figure, and Pressey and Smith (1986) failed to find unequivocal evidence for contrast in the Baldwin illusion. Of course, since the shortest test line used in the present experiments was $12.5 \%$ of the width of the figure, the occurrence of contrast with shorter test lines cannot be excluded. Nevertheless, the present finding reinforces Pressey's (1988) claim that the phenomenon of contrast is not well established, and that a major concern is to determine whether or not contrast can be reliably determined in visual illusion tasks.

\section{REFERENCES}

Brigell, M., \& UHLARIK, J. (1979). The relational determination of length illusions and length aftereffects. Perception, 8, 187-197.

Brigell, M., Uhlarik, J., \& Goldhorn, P. (1977). Contextual influences on judgments of linear extent. Journal of Experimental Psychology: Human Perception \& Performance, 3, 105-118.

FelLows, B. J. (1967). Reversal of the Müller-Lyer illusion with changes in the length of the inter-fins line. Quarterly Joumal of Experimental Psychology, 19, 208-214.

Fellows, B. J. (1968). The reverse Müller-Lyer illusion and 'enclosure.' British Journal of Experimental Psychology, 59, 369-372.

Fellows, B. J. (1973). Comments on assimilation theory and the reversed Müller-Lyer illusion. Perception, 2, 219-223.

Gillam, B., \&hambers, D. (1985). Size and position are incongruous: Measurements on the Müller-Lyer figure. Perception \& Psyqhophysics, 37, 549-556.

JoRDAN, K., UHLARIK, J. (1986). Length contrast in the Müller-Lyer figure: Functional equivalence of temporal and spatial separation. Perception \& Psychophysics, 39, 267-274.

KolberT, J., \& SARRIS, V. (1984). Illusional effects of spatial distance between focal and contextual extents of a modified Müller-Lyer figure. Perceptual \& Motor Skills, 59, 88-90.

KünNAPAs, T. M. (1955). Influence of frame size on apparent length of a line. Journal of Experimental Psychology, 50, 168-170.

Mack, A., Heuer, F., Villardi, K., \& Chambers, D. (1985). The dissociation of position and extent in Müller-Lyer figures. Perception \& Psychophysics, 37, 335-344.

Oyama, T. (1960). Japanese studies on the so-called geometrical optical illusions. Psychologia, 3, 7-20.

Predebon, J. (1983). Illusions of extent in simple angular figures. Perception, 12, 571-580.

Pressey, A. W. (1967). A theory of the Müller-Lyer illusion. Perceptual \& Motor Skills, 25, 569-572.

Pressey, A. W. (1988). A comment on "The effects of framing ratio and oblique length on Ponzo illusion magnitude." Perception \& Psychophysics, 43, 201-202.

Pressey, A. W., Bross, M. (1973). Assimilation theory and the reversed Müller-Lyer illusion. Perception, 2, 211-217.

Pressey, A. W., Di Lollo, V. (1978). Effects of distance between standard and comparison lines on the Müller-Lyer illusion. Perception \& Psychophysics, 24, 415-419.

Pressey, A. W., Di Lollo, V., \& TAit, R. (1977). Effects of gap size between shaft and fins and of angle of fins on the Müller-Lyer illusion. Perception, 6, 436-439.

Pressey, A. W., \& SmITh, N. E. (1986). The effects of location, orientation, and cumulation of boxes in the Baldwin illusion. Perception \& Psychophysics, 40, 344-350.

SCHIANo, D. J. (1986). Relative size and spatial separation: Effects on the parallel-lines illusion. Perceptual \& Motor Skills, 63, 1151-1155.

WORRALL, N., \& FIRTH, D. (1974). The components of the standard and reverse Müller-Lyer illusions. Quarterly Joumal of Experimental Psychology, 26, 342-354.

(Manuscript received August 19, 1991; revision accepted for publication March 17, 1992.) 